\title{
Designing a bunch compressor chicane for a multi-TeV linear collider
}

\author{
F. Stulle, ${ }^{*}$ A. Adelmann, and M. Pedrozzi \\ Paul Scherrer Institut, 5232 Villigen PSI, Switzerland
}

(Received 14 June 2006; published 20 March 2007)

\begin{abstract}
Incoherent and coherent synchrotron radiation are issues in most new accelerator projects utilizing bunch compressors to increase the peak current of the electron beam. Here we present a systematic approach to design a bunch compressor chicane suitable for the multi-TeV compact linear collider (CLIC). Based on analytical equations and beam dynamics simulations, we discuss how the influence of incoherent and coherent synchrotron radiation on the transverse beam emittance depends on chicane and electron beam parameters. The optimization of the bunch compressor layout and the electron beam parameters results in two different options which preserve the emittance to well within the specifications.
\end{abstract}

DOI: 10.1103/PhysRevSTAB.10.031001

PACS numbers: 29.27.Bd, 41.60.Ap

\section{INTRODUCTION}

The main beam line of the compact linear collider (CLIC) requires two bunch compressors to compress the electron bunches to an rms length of $30 \mu \mathrm{m}$. Such short bunches are a prerequisite at the interaction point to attaining the proposed high luminosity [1]. For the same reason, it is essential to maintain the transverse emittances during final bunch compression to within $\varepsilon_{n, x}<600 \mathrm{~nm} \mathrm{rad}$ and $\varepsilon_{n, y}<5 \mathrm{~nm} \mathrm{rad}$.

To determine suitable layouts for the bunch compressor chicanes and suitable electron beam parameters, it is necessary to analyze phenomena that induce emittance growth and consequently a reduction in luminosity. In this paper we focus on a systematic study of incoherent synchrotron radiation (ISR) and coherent synchrotron radiation (CSR) effects in the second bunch compressor chicane (BC2), which reduces the bunch length from $250 \mu \mathrm{m}$ to $30 \mu \mathrm{m}$. We discuss the relevant parameters and give a detailed description of our methodology which can be used as a general guideline for other bunch compressor designs.

The chicane parameters, whose effects on the performance of the bunch compressor are discussed, are total chicane length $L_{\text {tot }}$, dipole length $L_{\mathrm{B}}$, momentum compaction factor $R_{56}$, and dipole position. The influence of electron energy $E_{0}$, energy spread $\frac{\sigma_{E}}{E_{0}}$, initial beta function $\beta_{x, \text { ini }}$, and initial $\alpha_{x, \text { ini }}$ is also discussed. Parameter scans are performed for the dipole position, the initial beta function, and the initial alpha.

The chicanes studied here divide into two groups: Cchicanes with four dipoles [Fig. 1(a)] and S-chicanes with six dipoles [Fig. 1(b)]. The chicanes are referred to as symmetric in cases where the dipoles are of equal strength and the first and last drift are of equal length. Otherwise they are referred to as asymmetric.

Electrons traversing a dipole magnet emit synchrotron radiation mainly in the direction of motion $[2,3]$ resulting

\footnotetext{
*Electronic address: frank.stulle@psi.ch
}

in a change in electron energy. Because of the dispersive coupling in the bunch compressor chicane the energy change will, in turn, influence the horizontal, i.e., bending plane, phase space distribution. This effect is much stronger than the direct emittance dilution induced by the horizontal component of the synchrotron radiation fields. Since there is no vertical dispersion and the vertical field component is even weaker than the horizontal one, the impact of ISR and CSR in the vertical plane is negligible. The beam dynamics in the vertical phase space is therefore not discussed here.

Additionally, we neglect in our analysis the influence of the conducting walls of the vacuum chamber. The induced wakefields tend to enlarge the emittance (e.g. see [4]), the shielding effect reduces the CSR power [5]. This is done as we aim to optimize the parameters so that $\mathrm{BC} 2$ does not rely on shielding to reach the emittance goal. Consequently, the vacuum chamber can be wide enough to reduce wakefields to an acceptable level.

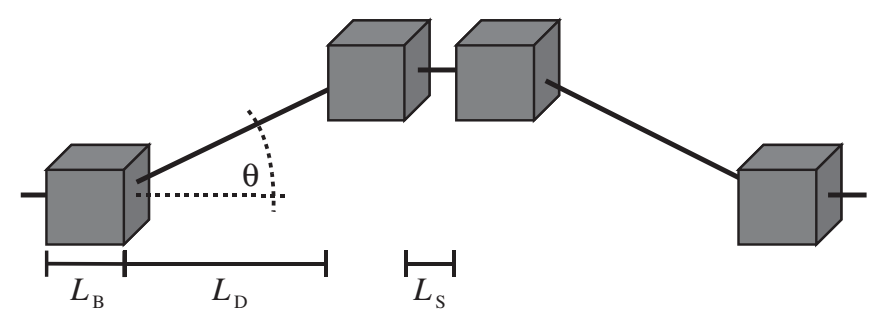

(a)

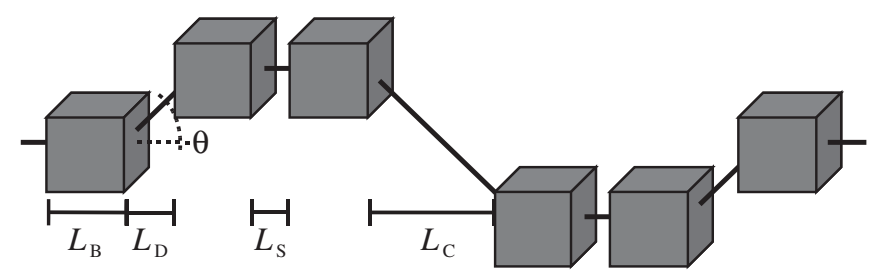

(b)

FIG. 1. C-chicane (a) and S-chicane (b). 
The next section discusses the choice of some basic chicane and electron beam parameters. Sections III and IV review the impact of incoherent and coherent synchrotron radiation on the emittance and how it is influenced by the dipole length. Section V describes the parameter scans. Some general remarks on the computer simulations and the computer code are given in Sec. VI. Section VII compares the results of the parameter scans.

\section{PARAMETERS OF THE CHICANES AND THE ELECTRON BEAM}

The choice of $L_{\text {tot }}$ is based on a proposal for $\mathrm{BC} 2$ given in [6]. There a C-chicane is proposed with a total length of $30 \mathrm{~m}$. It is built of four equal dipoles of $7 \mathrm{~m}$ length. While the ISR emittance growth in this chicane would be only about $1 \mathrm{~nm}$ rad, beam dynamics simulations show that the horizontal normalized emittance would grow by more than $50 \%$ due to CSR, when neglecting the shielding effect of the vacuum chamber. Elongating this chicane to $L_{\text {tot }}=$ $40 \mathrm{~m}$ reduces the CSR emittance growth to about $10 \%$, i.e., a value which is only a factor of 2 higher than the allowed emittance growth. A further reduction of the CSR emittance growth is achieved by shortening the dipoles. Regarding the choice of $L_{\mathrm{B}}=2 \mathrm{~m}$ refer to Secs. III and IV.

To reach a final bunch length of $\sigma_{s, f}=30 \mu \mathrm{m}$ while keeping the total energy spread below $2 \%$ [1], a combination of momentum compaction factor $R_{56}=-14 \mathrm{~mm}$ and linear position-energy correlation $u=\frac{1}{E_{0}} \frac{d E}{d s}=-70.5 \mathrm{~m}^{-1}$ along the electron bunches is needed. Lowering the $R_{56}$ would require a too high energy spread and enlarging the $R_{56}$ would lead to too long bunches since to first order

$$
\sigma_{s, f}=\sqrt{\left(1-R_{56} u\right)^{2} \sigma_{s, i}^{2}+R_{56}^{2}\left(\frac{\sigma_{E, \text { unc }}}{E_{0}}\right)^{2}} .
$$

Already with $R_{56}=-14 \mathrm{~mm}$ the uncorrelated energy spread of $\frac{\sigma_{E \text {,unc }}}{E_{0}}=2 \times 10^{-3}$ limits the minimum achievable bunch length to just below $30 \mu \mathrm{m}$. That also means, even with the given parameters, the bunches have to be fully compressed in $\mathrm{BC} 2$.

The linear position-energy correlation, called energy chirp, of $\frac{1}{E_{0}} \frac{d E}{d s}=-70.5 \mathrm{~m}^{-1}$ has to be imposed on the electron bunches by the rf cavities preceding the bunch compressor chicane. Additionally, the rf will add nonlinear correlations depending on the frequencies and phases of the cavities. In our case, this effect can be neglected since the shape of the longitudinal profile of the compressed bunch is dominated by the high uncorrelated energy spread. Note that this is in contrast to longitudinal beam dynamics in a free-electron laser. There the uncorrelated energy spread is very small, the nonlinearities can dominate, and a very short spike in the beam profile can develop, which emits huge CSR power (e.g. see [7]). In any case, the accelerator preceding $\mathrm{BC} 2$ will be designed to minimize the rf nonlinearities imposed on the longitudinal phase space distribution.

The beam energy of $E_{0}=9 \mathrm{GeV}$, which is chosen for the location of BC2, is a trade-off between ISR and CSR emittance growth. ISR would favor lower beam energies since the ISR radiation power is $P_{\mathrm{ISR}} \propto E_{0}^{4}$ [8] and CSR would favor higher beam energies since $P_{\mathrm{CSR}}$ is independent of the beam energy [5], but a higher energy beam is more rigid with respect to the forces acting on it.

It is expected that the initially Gaussian phase space distribution accumulates some distortions in the damping rings due to collective effects [9] but full-featured beam dynamics simulations are not yet finished [10]. Therefore, in all simulations presented here the transverse and longitudinal phase space distributions are assumed to be Gaussian. This assumption can be justified as long as the realistic longitudinal profile is well behaved, i.e., it initially contains no high current spikes. In this case, it is impossible for such spikes to develop in the bunch compressor due to the high uncorrelated energy spread. For example, an initially uniform profile would lead only to a longer final bunch and thus to a reduction of CSR. Again, this is in contrast to projects where the beam has a small uncorrelated energy spread, e.g., FELs. There a lot more care has to be taken when modeling the longitudinal profile because sharp spikes might develop during compression (e.g. see [7]).

Parameters common to all chicanes can be found in Table I. The corresponding initial electron beam parameters are given in Table II. It is specified that after compression horizontal and vertical emittance should not exceed $\varepsilon_{n, x}=600 \mathrm{~nm} \mathrm{rad}$ and $\varepsilon_{n, y}=5 \mathrm{~nm} \mathrm{rad}$, respectively.

TABLE I. Parameters common to all bunch compressor chicanes compared in this paper.

\begin{tabular}{lccc}
\hline \hline & Symbol & Value & Unit \\
\hline Total length & $L_{\text {tot }}$ & 40.0 & $\mathrm{~m}$ \\
Dipole length & $L_{\mathrm{B}}$ & 2.0 & $\mathrm{~m}$ \\
Dipole pair separation & $L_{\mathrm{S}}$ & 1.0 & $\mathrm{~m}$ \\
Momentum compaction factor & $R_{56}$ & -0.014 & $\mathrm{~m}$ \\
\hline \hline
\end{tabular}

TABLE II. Parameters of the electron bunch in front of the bunch compressor chicane.

\begin{tabular}{lccc}
\hline \hline & Symbol & Value & Unit \\
\hline Beam energy & $E_{0}$ & 9 & $\mathrm{GeV}$ \\
Bunch charge & $Q_{0}$ & 0.41 & $\mathrm{nC}$ \\
Bunch length & $\sigma_{s, i}$ & 250 & $\mu \mathrm{m}$ \\
Uncorrelated energy spread & $\frac{\sigma_{E, \text { unc }}}{E_{0}}$ & $2 \times 10^{-3}$ & \\
Linear $s$ - $E$ correlation & $u=\frac{1}{E_{0}} \frac{d E}{d s}$ & -70.5 & $\mathrm{~m}^{-1}$ \\
Normalized emittance & $\varepsilon_{n, x}$ & 570 & $\mathrm{~nm} \mathrm{rad}$ \\
& $\varepsilon_{n, y}$ & 4 & $\mathrm{~nm} \mathrm{rad}$ \\
\hline \hline
\end{tabular}




\section{INCOHERENT SYNCHROTRON RADIATION}

The growth of the normalized emittance induced by ISR can be analytically estimated by using the equation

$$
\Delta \varepsilon_{n, x} \approx 8 \times 10^{-8} E_{0}^{6} \frac{\theta^{5}}{L_{\mathrm{B}}^{2}}\left(L_{\mathrm{D}}+L_{\mathrm{B}}+\frac{\beta_{\min }+\beta_{\max }}{3}\right)
$$

which was derived in [8]. Here $E_{0}$ is the energy in units of $\mathrm{GeV}$. The dipoles are of length $L_{\mathrm{B}}$ and deflect the electrons by an angle $\theta . L_{\mathrm{D}}$ is the length of the drift between the first and the second dipole. $\beta_{\min }$ and $\beta_{\max }$ are minimum and maximum beta functions along the chicane. Equation (2) is an approximation of

$$
\Delta \varepsilon_{n, x}=4 \times 10^{-8} E_{0}^{6} I_{5}
$$

for the case of a symmetric C-chicane with small bending angles and symmetric beta functions. Using these assumptions, the fifth synchrotron radiation integral $I_{5}$ can be simplified [11].

To study the effect of ISR in a symmetric C-chicane, the length of the dipole magnets $L_{\mathrm{B}}$ is varied while the total chicane length $L_{\mathrm{tot}}$, the separation of the central dipoles $L_{\mathrm{S}}$, and the $R_{56}$ are kept constant (Fig. 2). Note that the bending angle $\theta$ is not constant, but rather a function to first order of the aforementioned parameters:

$$
\theta \approx \sqrt{\frac{-R_{56}}{L_{\mathrm{tot}}-\frac{8}{3} L_{\mathrm{B}}-L_{\mathrm{S}}}} .
$$

Consequently, the bending angle slowly drops for decreasing dipole length. For each $L_{\mathrm{B}}$, the ISR emittance growth is calculated by numerically integrating Eq. (3). It is found that for dipole lengths down to about $L_{\mathrm{B}}=4 \mathrm{~m}$ the emittance growth is almost constant. For dipoles shorter than $4 \mathrm{~m}$ it gets stronger, but even for $L_{\mathrm{B}}=1.5 \mathrm{~m}$ it is still below $1 \%$.

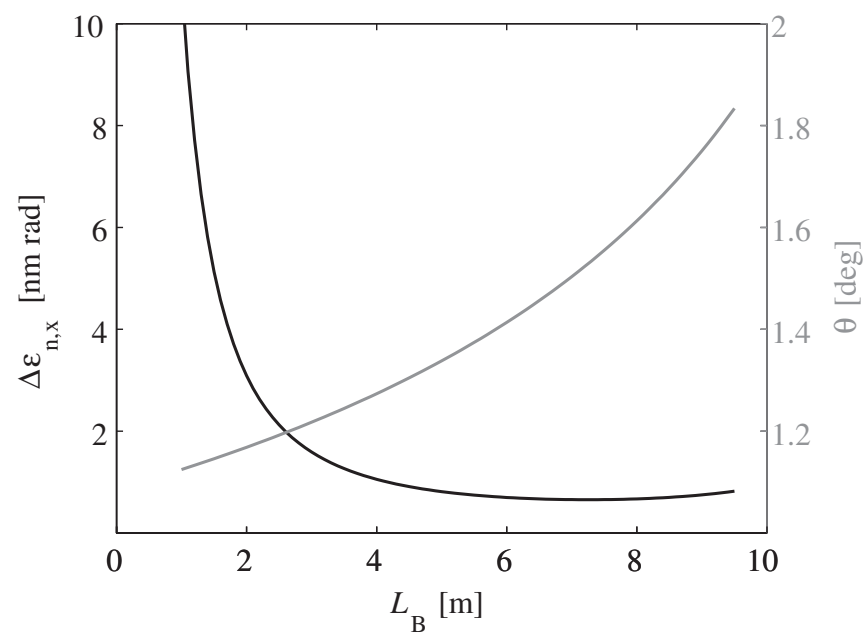

FIG. 2. Variation of ISR emittance growth (black, left axis) and bending angle (gray, right axis) with dipole length.
This behavior of the emittance growth can be explained because $\Delta \varepsilon_{\mathrm{n}, \mathrm{x}} \propto \theta^{5} / L_{\mathrm{B}}^{2}$. That means the variation of the bending angle counteracts the influence of the dipole length. Consequently, within certain boundaries it does not matter for the ISR emittance growth if longer or shorter dipoles are used. The boundaries are of course not universal but depend on chicane parameters. For the parameters of $\mathrm{BC} 2$, it is feasible to reduce the dipole length to about $L_{\mathrm{B}}=2 \mathrm{~m}$ without adversely affecting the emittance.

\section{COHERENT SYNCHROTRON RADIATION}

From synchrotron radiation theory, it is known that the coherently radiated power scales as $P_{\mathrm{CSR}} \propto R^{-2 / 3}$ [5], where $R$ is the bending radius. Therefore one could expect that with respect to the CSR emittance growth it would be of advantage to choose long dipoles, i.e., large bending radii, to reduce $P_{\mathrm{CSR}}$. On the other hand, the motion of the electrons through a bunch compressor is not circular but consists of several arcs. Hence, the steady-state approach as used in Ref. [5] is not applicable.

This problem has been addressed in Ref. [12]. An analysis of the radiation of an electron bunch traversing a dipole magnet is performed taking transient effects into account. It is shown that the total energy loss due to CSR of an electron bunch with rectangular profile traversing a single dipole is given by

$$
\begin{aligned}
\Delta E_{\mathrm{tot}}= & \left(\frac{3^{2 / 3} N_{e}^{2} e_{0}^{2}}{4 \pi \varepsilon_{0} R^{2 / 3} \sigma_{s}^{4 / 3}}\right) R \theta\left\{1+\frac{3^{1 / 3} 4}{9} \frac{\sigma_{s}^{1 / 3}}{R^{1 / 3} \theta}\right. \\
& \left.\times\left[\ln \left(\frac{\sigma_{\mathrm{s}} \gamma^{3}}{R}\right)-4\right]\right\} .
\end{aligned}
$$

Obviously, the dependence on the bending radius $R$ is more complex than in the steady-state theory. Depending on the values of bunch length $\sigma_{s}$, relativistic factor $\gamma$, and bending angle $\theta$, it is possible to reduce the energy loss by reducing $R$, i.e., by using short dipoles. One also has to keep in mind that the use of shorter dipoles leads to a reduction of the bending angle $\theta$, as we have seen in the last section. Consequently, it can be argued that the emittance growth induced by CSR should drop for chicanes built of shorter dipoles.

Indeed, this is the case for the $\mathrm{BC} 2$ parameters as can be seen in Fig. 3. It compares the relative energy loss $\frac{\Delta E_{\mathrm{tot}}}{E_{\mathrm{tot}}}$ calculated using Eq. (4) for a $100 \mu \mathrm{m}$ long bunch traversing a single dipole of $\mathrm{BC} 2$ to results of beam dynamics simulations including CSR for the case of a symmetric Cchicane. The growth of the projected emittance increases by about a factor of 2 when increasing the dipole length from $1 \mathrm{~m}$ to $9.5 \mathrm{~m}$. The dependence of $\frac{\Delta E_{\mathrm{tot}}}{E_{\mathrm{tot}}}$ on the dipole length is similar and consequently Eq. (4) is very useful when designing a bunch compressor chicane.

In contrast to the projected emittance, the slice emittance growth is almost constant. The reason is that the 


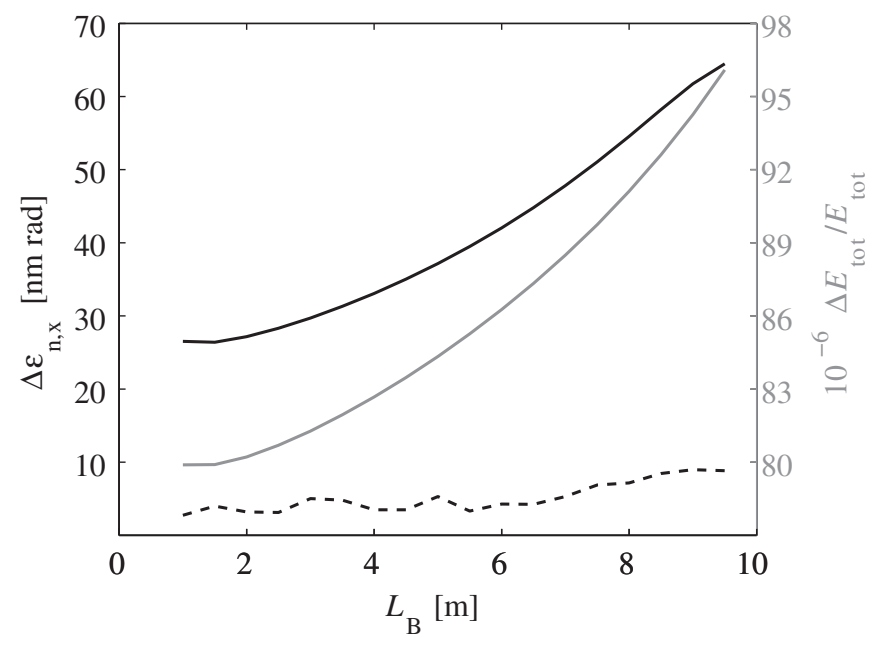

FIG. 3. Variation of projected (solid black) and slice (dashed black) CSR emittance growth with dipole length. As a comparison, the relative energy loss of a $100 \mu \mathrm{m}$ long bunch traversing a single dipole of BC2 is also plotted (gray, right axis).

phase space coordinates of all electrons at the same longitudinal position inside the electron bunch are affected in a similar way due to the energy change induced by CSR. This effect is usually called correlated emittance growth and is the main effect of CSR.

The simulations are performed with the computer code CSRTRACK [13] and make use of a CSR model based on the theory derived in [14]. A general discussion of the computer simulations is given in Sec. VI.

\section{DESCRIPTION OF THE PARAMETER SCANS}

Within the framework of the chicane and electron beam parameters given, a number of free parameters remain to find suitable solutions for the bunch compressor chicane and the corresponding electron beam.

The performance of a bunch compressor strongly depends on the positions of the dipoles with respect to each other. Of course, the final dispersion should always vanish and the total length of all chicanes compared should be the same. The positions of the first and the last dipole are therefore fixed.

Additionally, it is assumed that the dispersion in the drift between the two central dipoles in a C-chicane is constant. The length of this drift has no influence on bunch compression and should be kept short. A constant value of $L_{\mathrm{S}}=1 \mathrm{~m}$ was chosen to allow the installation of diagnostics, collimation, etc. Consequently, only 2 degrees of freedom, i.e., longitudinal and horizontal position of the central dipole pair, are left for the parameter scans. One is needed to correct the $R_{56}$ which should also be kept constant. In the S-chicanes the separation of the second and third as well as the separation of the fourth and fifth dipole is kept constant. Hence, longitudinal and horizontal positions of two dipole pairs can be scanned. Again one of the 4 degrees of freedom is needed to correct the $R_{56}$. It is important to note that S-chicanes are never fully achromatic, but the amount of the residual dispersion can be made sufficiently small to be neglected [15].

The advantage of shifting the central dipoles towards the front of a bunch compressor chicane is that one increases the synchrotron radiation power when the electron bunches are still long and reduce it when the bunches are short. Because of the nonlinear dependence of CSR power on bending angle and bunch length [12], this can lead to an overall reduction of the radiation power and the emittance dilution induced by CSR.

In S-chicanes an additional effect has to be considered. They introduce a partial geometric compensation of the impact of CSR on the horizontal phase space distribution, similar to the case of double C-chicanes intersected by a $-I$-transformation of the transverse phase space coordinates [16]. This compensation can be optimized by shifting the central four dipoles closer to the end of the chicane without changing their bending angles [15].

Besides geometric parameters, also the initial values of the optics functions $\beta_{x \text {,ini }}$ and $\alpha_{x}$,ini have a strong influence on the growth of the projected emittance imposed by CSR

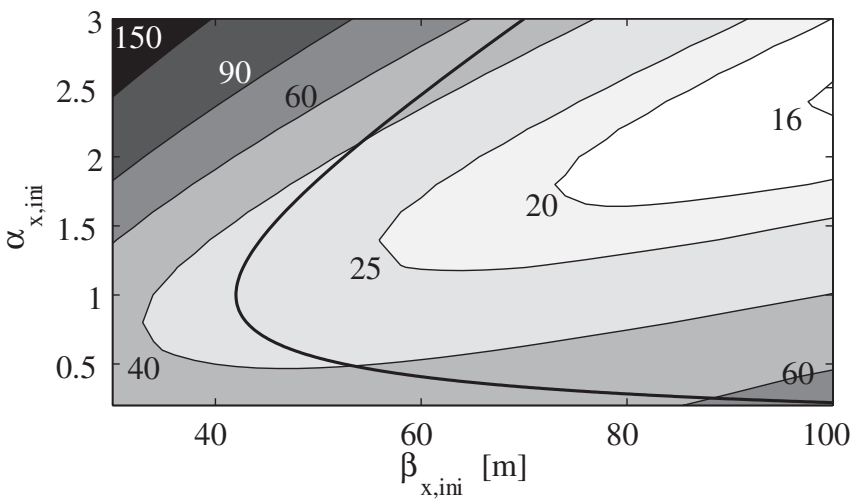

(a)

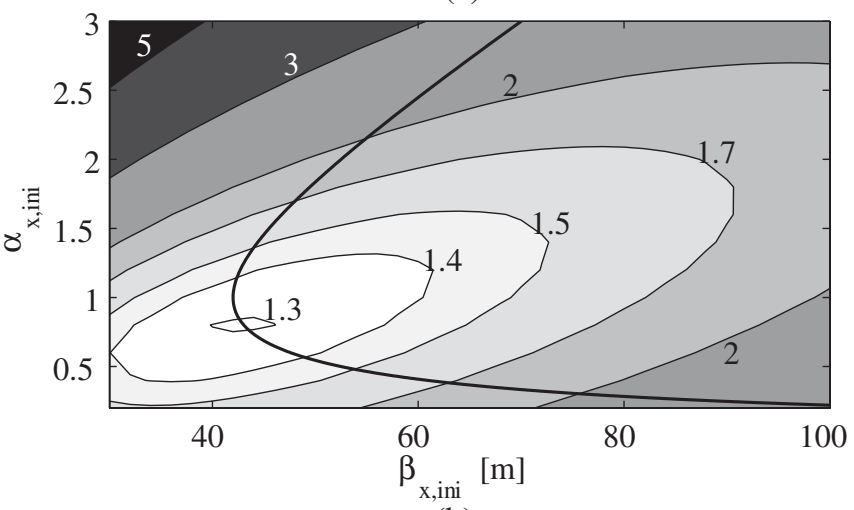

(b)

FIG. 4. Contour plots of CSR (a) and ISR (b) emittance growth versus initial beta function and alpha. The thick black lines mark the initial values resulting in symmetric beta functions. Values on the left side of these lines result in a waist of the beta function closer to the front of the chicane, values on the right side result in a waist closer to the end. 


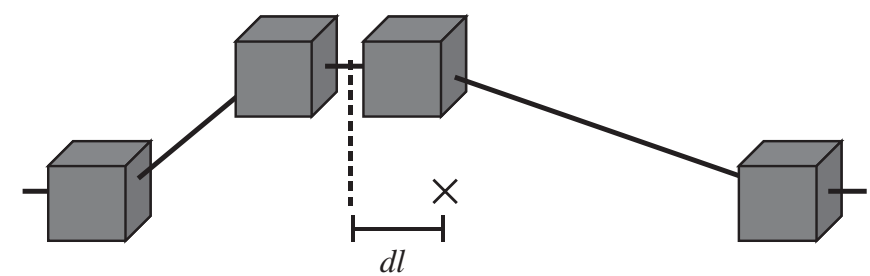

(a)

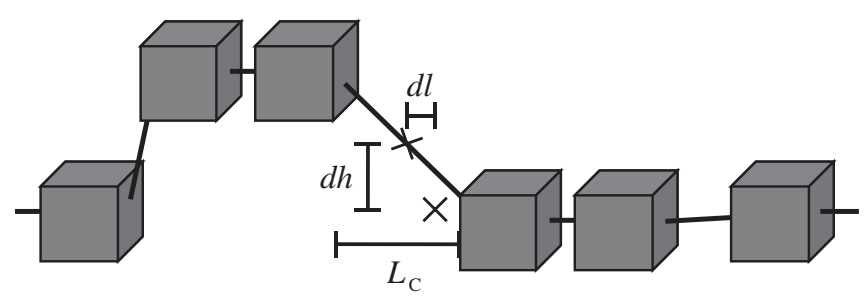

(b)

FIG. 5. Free geometric parameters in the scans of the Cchicane (a) and S-chicane (b).

[17]. Figure 4 shows results of beam dynamics simulations of a symmetric C-chicane with varying $\beta_{x \text {,ini }}$ and $\alpha_{x \text {,ini }}$. For the CSR emittance growth, a waist of the beta function close to the last dipole of the chicane is usually preferable [Fig. 4(a)]. In contrast to this ISR favors beta functions symmetric along the chicane and small average values of the beta function [Fig. 4(b)].

In total we have to scan three parameters for the Cchicane and five parameters for the S-chicane. For the $\mathrm{C}$-chicanes these parameters are the longitudinal position $d l$ of the central pair of dipoles measured with respect to their position in the symmetric C-chicane [Fig. 5(a)] and the initial optics functions $\beta_{x, \text { ini }}$ and $\alpha_{x, \text { ini }}$. The horizontal position of the dipole pair is used to correct the $R_{56}$. In the S-chicane besides $\beta_{x, \text { ini }}$ and $\alpha_{x, \text { ini }}$ three geometric parameters are scanned. These are the length of the central drift $L_{\mathrm{C}}$ and the longitudinal and horizontal shifts $d l$ and $d h$ of the midpoint of the two dipole pairs with respect to its position in the symmetric S-chicane [Fig. 5(b)]. The horizontal distance of the dipole pairs is used to correct the $R_{56}$.

\section{GENERAL REMARKS ON COMPUTER SIMULATIONS}

All simulations were performed with the code CSRTRACK [13]. In this code several different methods for the calculation of the CSR fields are implemented. They can be divided into three groups: three-dimensional integration of the electromagnetic fields, two-dimensional pseudo-Green's function approach, and calculation of one-dimensional analytical formulas.

The first method calculates longitudinal, horizontal, and vertical radiation field components. Its computational effort scales with the square of the number of particles $N$. It is therefore too slow for making parameter scans even on

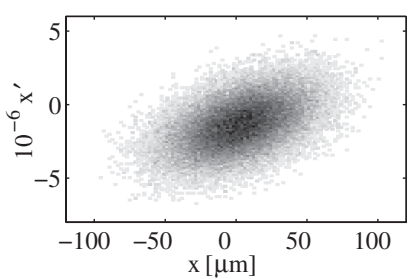

(a)

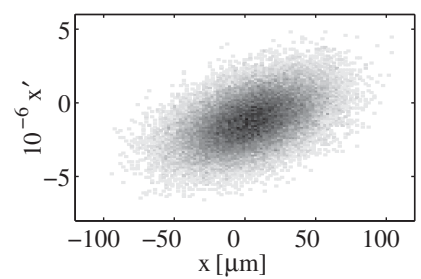

(b)
FIG. 6. Horizontal phase space distributions behind a symmetric $\mathrm{C}$-chicane obtained with the one-dimensional method (a) and the two-dimensional method (b). The corresponding normalized emittances are $587.2 \mathrm{~nm}$ rad and $586.3 \mathrm{~nm} \mathrm{rad}$, respectively.

parallel computers. The second method calculates only the longitudinal and horizontal field components making the computational effort smaller by about 2 orders of magnitude, but the scaling with $N^{2}$ remains. Only the last method, which calculates the longitudinal radiation field component from analytic formulas, is fast enough for extensive parameter scans. For a single simulation about 30 min of CPU time were needed. The results presented in the next section are a summary of many thousand simulations.

The code CSRTRACK uses small Gaussian charge distributions, so called sub-bunches, to model the charge distribution of an electron bunch. They are tracked selfconsistently through the beam line taking the electromagnetic fields radiated by the sub-bunches into account. Another set of pointlike test particles can be tracked inside the fields of the sub-bunches, but they do not add to the electromagnetic radiation.

Details on the calculation methods used in CSRTRACK can be found in $[13,14,18]$. A general overview of CSR codes and the various approaches used therein to calculate CSR is given in [19]. Several codes and models have been compared in a benchmark test [20,21], where it is concluded that in many cases the simple one-dimensional CSR calculation is sufficient for the design of a bunch compressor chicane.

A comparison of simulation results obtained for a symmetric C-chicane with the two-dimensional and the onedimensional method is shown in Fig. 6. One can see that the final horizontal phase space distributions are almost the same. The corresponding projected emittances are $587.2 \mathrm{~nm} \mathrm{rad}$ for the one-dimensional method and $586.3 \mathrm{~nm} \mathrm{rad}$ for the two-dimensional method. These values are in very good agreement, justifying the use of the one-dimensional method in the computer simulations to design $\mathrm{BC} 2$.

\section{RESULTS OF THE PARAMETER SCANS}

For the comparison of the results from the various parameter scans, only the emittance growth due to ISR and CSR is considered as the variation of other electron beam parameters is too small to be of importance. 


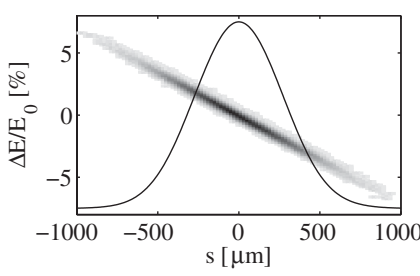

(a)

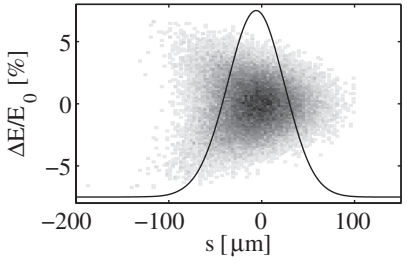

(c)

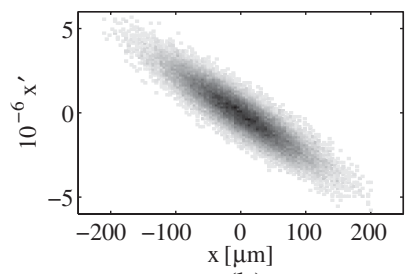

(b)

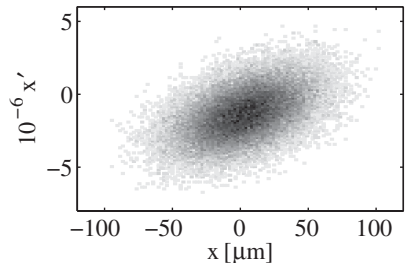

(d)
FIG. 7. On the left side plots of initial (a) and final (c) longitudinal phase space distributions and the corresponding charge profiles (a.u.) are shown. On the right side plots of initial (b) and final (d) horizontal phase space distributions are shown.

Figures 7(a) and 7(b) show examples of the initial longitudinal and horizontal phase space distributions. The distributions behind a symmetric $\mathrm{C}$-chicane are shown in Figs. 7(c) and 7(d). The initial longitudinal phase space distribution was the same for all parameter scans. The final longitudinal phase space distribution, which is similar for all chicanes compared, is slightly influenced by the second order transfer matrix element $T_{566}$. But the shape of the charge profile is fully dominated by the Gaussian uncorrelated energy spread. There is no impact of CSR visible.

Since in the scans the initial beta function and the alpha are varied, the shapes of the initial horizontal phase space distributions vary, but the initial emittances were always the same. The final emittances of course differ. Another effect imposed by the CSR energy loss is that the mean values of the horizontal phase space coordinates $\bar{x}$ and $\bar{x}^{\prime}$ are not exactly zero [Fig. 7(d)].

In a first parameter scan for the C-chicane only the position of the central dipole pair was changed. The initial optics functions were chosen as $\beta_{x, \text { ini }}=45 \mathrm{~m}$ and $\alpha_{x \text {,ini }}=$ 1.45. These values result in a symmetric $\beta_{x}$ along the chicane and a small average $\beta_{x}$. In this case ISR and CSR emittance growth is already small (Fig. 8). Here the minimum emittance growth is $\Delta \varepsilon_{n, x}=30 \mathrm{~nm} \mathrm{rad}$, i.e., a value at the upper limit of what is allowed by the specifications. The best layout in this scan is an asymmetric C-chicane where the central dipoles are shifted by about $d l=3 \mathrm{~m}$ towards the front of the chicane.

The full three-dimensional scan of $d l, \beta_{x, \text { ini }}$, and $\alpha_{x, \text { ini }}$ results in a minimum emittance growth of $\Delta \varepsilon_{n, x}=$ $12 \mathrm{~nm} \mathrm{rad}$. The dependence of the emittance growth on $d l$ and $\beta_{x, \text { ini }}$ is summarized in Fig. 9. For each point in the plot the value for the optimum $\alpha_{x, \text { ini }}$ is chosen. It is interesting to note that the best values found are for an asymmetric $\mathrm{C}$-chicane where the central dipoles are shifted

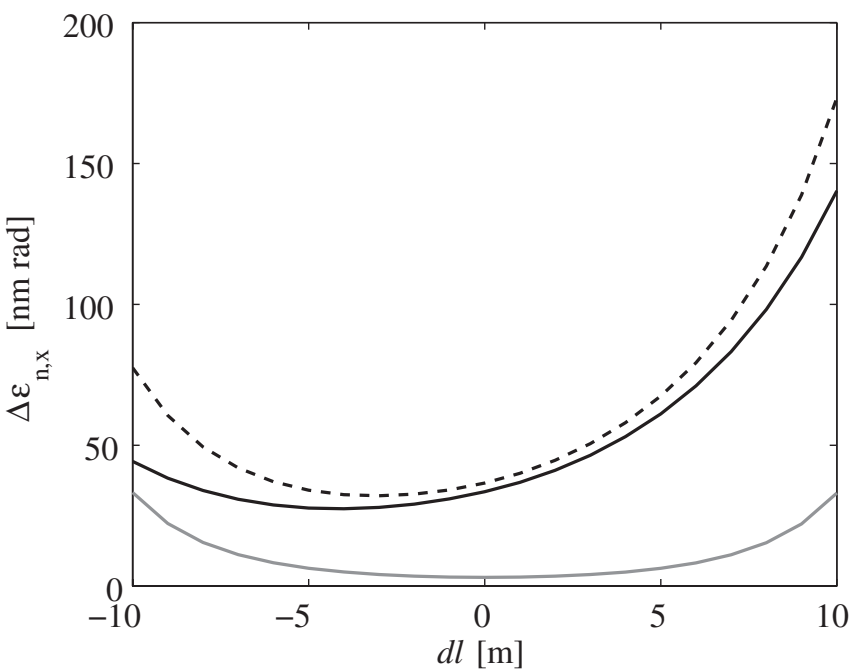

FIG. 8. Variation of ISR (gray), CSR (black), and ISR + CSR (dashed) emittance growth with the position of the central dipoles in a $\mathrm{C}$-chicane.

by $2 \mathrm{~m}$ towards the end of the chicane. This behavior contradicts the expectation that it should be preferable to shift the dipoles towards the front of the chicane (see Sec. V). Nevertheless, the symmetric C-chicane with optimized initial optics functions $\beta_{x, \text { ini }}=200 \mathrm{~m}$ and $\alpha_{x, \text { ini }}=$ 4.8 already results in an emittance growth of $\Delta \varepsilon_{n, x}=$ $14 \mathrm{~nm} \mathrm{rad}$ which is only about half the allowed value.

As already mentioned, in an S-chicane there are more degrees of freedom for the layout. How the emittance growth depends on the length of the central drift $L_{\mathrm{C}}$ is plotted in Fig. 10. For each $L_{\mathrm{C}}$, the other two parameters, $d l$ and $d h$, are scanned to find the minimum values of ISR emittance growth, CSR emittance growth, and combined ISR plus CSR emittance growth. The lowest value found is $\Delta \varepsilon_{n, x}=10.5 \mathrm{~nm}$ rad for $L_{\mathrm{C}}=11 \mathrm{~m}$. In a symmetric $\mathrm{S}$ -

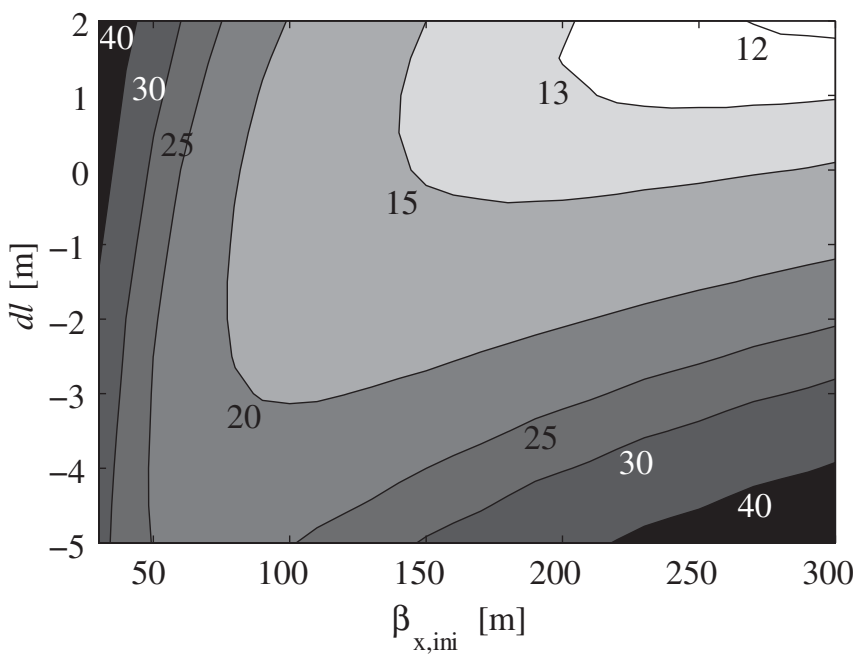

FIG. 9. Contour plot of the emittance growth in a C-chicane versus dipole shift $d l$ and initial beta function $\beta_{x, \text { ini }}$. 


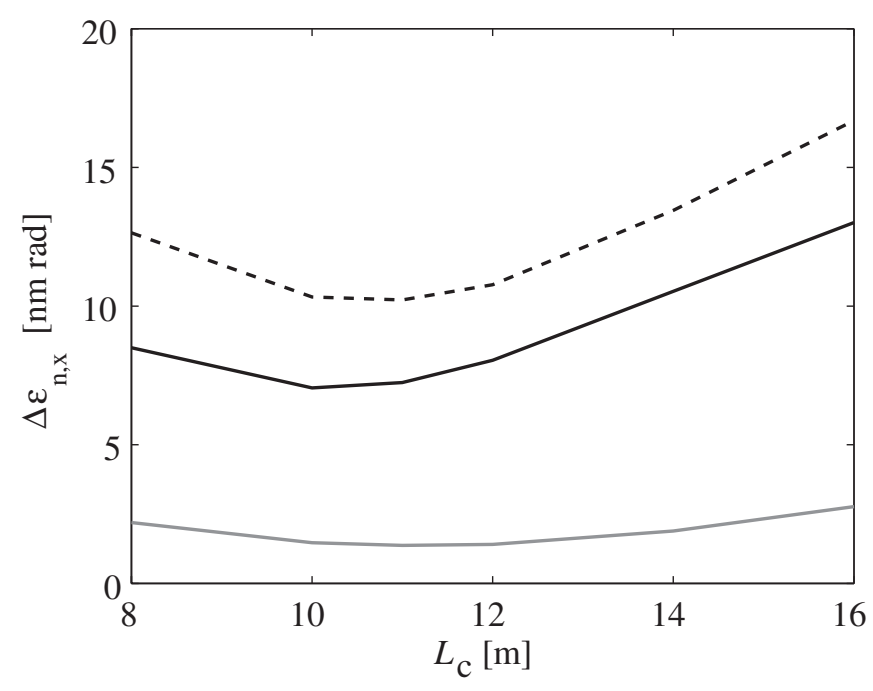

FIG. 10. Variation of minimum ISR (gray), CSR (black), and ISR + CSR (dashed) emittance growth with the length of the central drift of an S-chicane.

chicane there is $L_{\mathrm{C}} \approx 14 \mathrm{~m}$. The initial optics functions were chosen as $\beta_{x, \text { ini }}=45 \mathrm{~m}$ and $\alpha_{x, \text { ini }}=1.45$.

The dependence of the emittance growth on a longitudinal and horizontal dipole shift $d l$ and $d h$ can be seen in Fig. 11. The minimum emittance growth of $\Delta \varepsilon_{n, x}=$ $10.5 \mathrm{~nm} \mathrm{rad}$ is achieved around $d l=0 \mathrm{~m}$ and $d h=$ $7.5 \mathrm{~cm}$. The above-mentioned initial optics functions were used and $L_{\mathrm{C}}=11 \mathrm{~m}$. Further scans including the optics functions showed that this value is already close to the optimum value of $\Delta \varepsilon_{n, x}=10 \mathrm{~nm} \mathrm{rad}$ obtained for $\beta_{x, \text { ini }}=52 \mathrm{~m}$ and $\alpha_{x, \text { ini }}=1.6$ (Fig. 12).

A comparison of the results for the symmetric C-chicane and the optimum S-chicane is given in Fig. 12. The corresponding chicane layouts are sketched in Fig. 13. It is

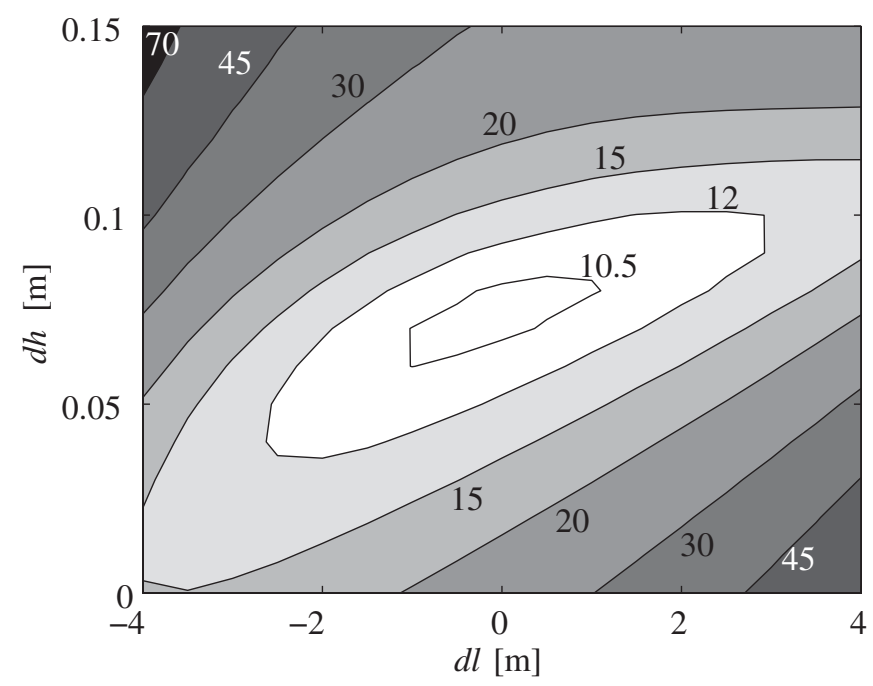

FIG. 11. Contour plot of the emittance growth versus longitudinal and horizontal dipole shift in an S-chicane with $L_{\mathrm{c}}=$ $11 \mathrm{~m}$.

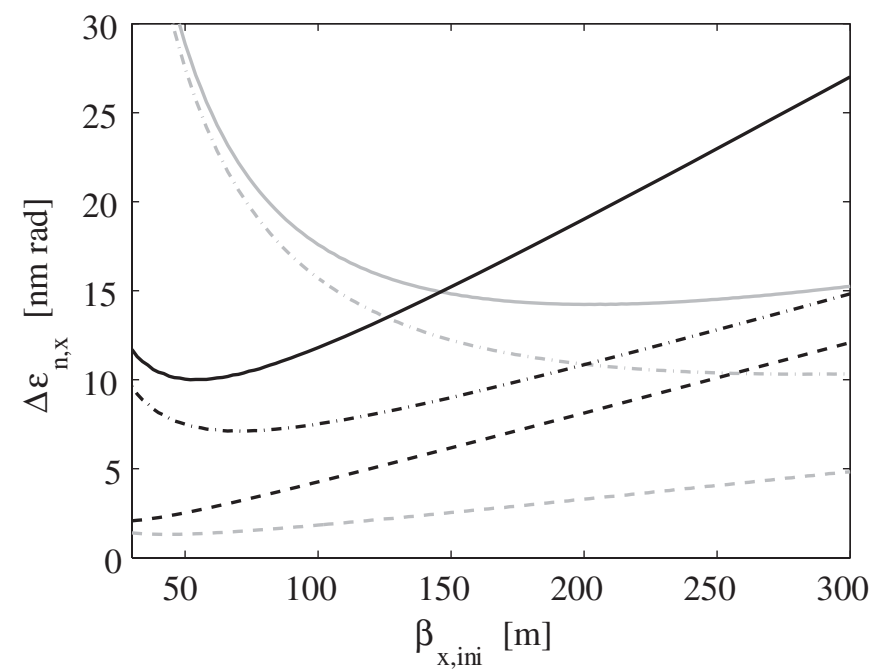

FIG. 12. ISR (dashed), CSR (dash-dotted), and ISR + CSR (solid) emittance growth in the optimized C-chicane (gray) and S-chicane (black) vs initial beta function. Only the values obtained for the optimum alpha are shown.

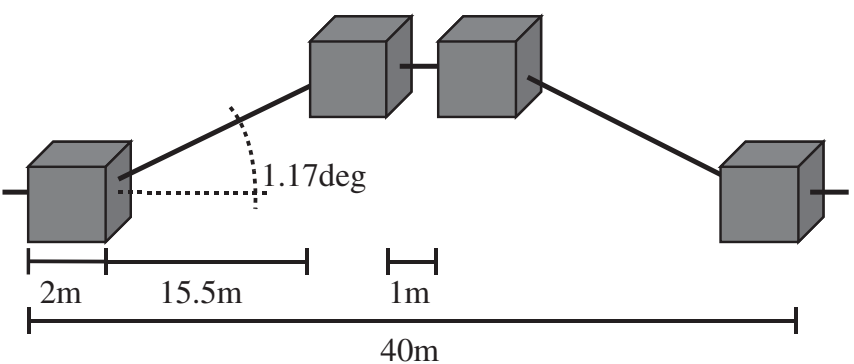

(a)

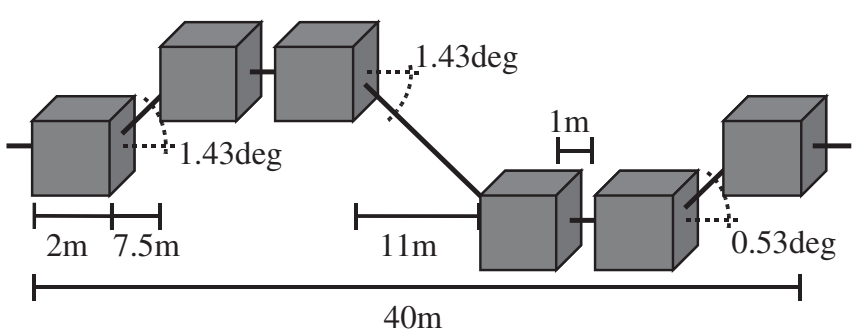

(b)

FIG. 13. Optimized geometries of the C-chicane (a) and the Schicane (b).

interesting to note that to reach the minimum emittance growth in the $\mathrm{C}$-chicane the initial beta function needs to be very high whereas in the S-chicane the optimum is reached for lower values of the beta function.

\section{CONCLUSION}

In this paper we systematically designed a bunch compressor chicane suitable for the multi-TeV linear collider CLIC. We discussed how the emittance growth induced by 
incoherent and coherent synchrotron radiation can be influenced by choosing the proper chicane and electron beam parameters and provide a guideline for the design of bunch compressor chicanes.

An important result is that the choice of the dipole length is crucial to building a bunch compressor chicane. Both too long and too short dipoles will lead to a strong emittance growth - in the first case due to CSR in the second due to ISR.

Of equal importance is the optimization of initial beam optics. A waist of the beta function closer to the end of the chicane will strongly reduce the emittance growth induced by CSR and will enhance the ISR emittance growth only weakly.

A consequence of our review of synchrotron radiation effects is that the chicane layout originally proposed for $\mathrm{BC} 2$ at CLIC [6], with its very long dipoles, is far from optimal. A chicane with the same $R_{56}$ but shorter dipoles and optimized beta functions is a better choice. Several different chicane layouts and sets of optics functions were systematically compared in various parameter scans. A $40 \mathrm{~m}$ long asymmetric S-chicane built of $2 \mathrm{~m}$ long dipoles was found to be the best solution in terms of ISR and CSR emittance growth. Nevertheless, a symmetric C-chicane of the same length built of the same dipoles also gives good results. Using optimum initial optics functions, the amount of emittance growth is $\Delta \varepsilon_{n, x}=14 \mathrm{~nm}$ rad for the symmetric $\mathrm{C}$-chicane and $\Delta \varepsilon_{n, x}=10 \mathrm{~nm}$ rad for the asymmetric $\mathrm{S}$-chicane. Both values are well within the specification of $\Delta \varepsilon_{n, x}<30 \mathrm{~nm}$ rad.

\section{ACKNOWLEDGMENTS}

This work is supported by the Commission of the European Communities under the 6th Framework Programme "Structuring the European Research Area," Contract No. RIDS-011899.
[1] G. Guignard et al., CERN 2000-008, 2000.

[2] J.D. Jackson, Classical Electrodynamics, Third Edition (John Wiley \& Sons, Inc., New York, 1999).

[3] G. Geloni et al., DESY 03-044, 2003.

[4] G. V. Stupakov, SLAC PUB-8683, 2000.

[5] J. S. Nodvick and D. S. Saxon, Phys. Rev. 96, 180 (1954).

[6] T. E. d'Amico, G. Guignard, and T. Raubenheimer, CERN CLIC Note-372, 1998.

[7] M. Dohlus and T. Limberg, Electron Bunch Length Compression, in ICFA Beam Dynamics Newsletter 38, 2005, pp. 15-37.

[8] T. Raubenheimer, P. Emma, and S. Kheifets, in Proceedings of PAC93, Washington D.C., 1993.

[9] M. Korostelev, CERN CLIC-Note-701, 2006.

[10] D. Schulte (private communication).

[11] R. H. Helm, M. J. Lee, P. L. Morton, and M. Sands, SLAC PUB-1193, 1973.

[12] E. L. Saldin, E. A. Schneidmiller, and M. V. Yurkov, Nucl. Instrum. Methods Phys. Res., Sect. A 398, 373 (1997).

[13] M. Dohlus and T. Limberg, in Proceedings of FEL04, Trieste, Italy, 2004.

[14] M. Dohlus, DESY TESLA-FEL 2003-05, 2003.

[15] F. Stulle, DESY Thesis-2004-041, 2004.

[16] P. Emma and R. Brinkmann, in Proceedings of PAC97, Vancouver, Canada, 1997.

[17] M. Dohlus and T. Limberg, in Proceedings of PAC05, Knoxville, Tennessee, 2005.

[18] E. L. Saldin, E. A. Schneidmiller, and M. V. Yurkov, Nucl. Instrum. Methods Phys. Res., Sect. A 417, 158 (1998).

[19] G. Bassi et al., Nucl. Instrum. Methods Phys. Res., Sect. A 557, 189 (2006).

[20] ICFA Beam Dynamics Mini-Workshop on CSR, BerlinZeuthen, 2002, see http://www.desy.de/csr

[21] Proceedings of the ICFA Workshop "The Physics and Applications of High Brightness Electron Beams," Chia Laguna, Sardinia, 2002, edited by J. Rosenzweig, G. Travish, and L. Serafini; see also http:// home.physics. ucla.edu/calendar/conferences/AABD/ 